\title{
Erratum to: Influence of 3d transition-metal substitution on the oxygen reduction reaction electrocatalysis of ternary nitrides in acid
}

Kevin E. Fritz ${ }^{1}$, Yichen Yan $^{1}$, and Jin Suntivich ${ }^{1,2}(\square)$

${ }^{1}$ Department of Materials Science and Engineering, Cornell University, Ithaca, New York 14850, USA

${ }^{2}$ Kavli Institute at Cornell for Nanoscale Science, Ithaca, New York 14850, USA

( Tsinghua University Press and Springer-Verlag GmbH Germany, part of Springer Nature 2020

\section{Erratum to}

Nano Research 2019, 12(9): 2307-2312

https://doi.org/10.1007/s12274-019-2440-6

The NSF number in Acknowledgements in page 2311 was unfortunately wrong,

\section{Instead of}

The work is supported by the National Science Foundation (NSF) under Grant No. CHE-1805400.

\section{It should read}

The work is supported by the National Science Foundation (NSF) under Grant No. CHE-1665305.

The online version of the original article can be found at https://doi.org/10.1007/s12274-019-2440-6 